\title{
Two-qutrit Entanglement Witnesses and Gell-Mann Matrices
}

\author{
M. A. Jafarizadeh ${ }^{a, b, c *}$, Y. Akbari ${ }^{a, b \dagger}$, N. Behzadi ${ }^{a, b} \ddagger$ \\ ${ }^{a}$ Department of Theoretical Physics and Astrophysics, Tabriz University, Tabriz 51664, Iran. \\ ${ }^{b}$ Institute for Studies in Theoretical Physics and Mathematics, Tehran 19395-1795, Iran. \\ ${ }^{c}$ Research Institute for Fundamental Sciences, Tabriz 51664, Iran.
}

November 4, 2018

*E-mail:jafarizadeh@tabrizu.ac.ir

${ }^{\dagger}$ E-mail:y-akbari@tabrizu.ac.ir

${ }^{\ddagger}$ E-mail:behzadi@tabrizu.ac.ir 


\begin{abstract}
The Gell-Mann $\lambda$ matrices for Lie algebra su(3) are the natural basis for the Hilbert space of Hermitian operators acting on the states of a three-level system(qutrit). So the construction of EWs for two-qutrit states by using these matrices may be an interesting problem. In this paper, several two-qutrit EWs are constructed based on the Gell-Mann matrices by using the linear programming (LP) method exactly or approximately. The decomposability and non-decomposability of constructed EWs are also discussed and it is shown that the $\lambda$-diagonal EWs presented in this paper are all decomposable but by adding $\lambda$-non-diagonal terms, one can obtain various non-decomposable EWs.

Keywords: Entanglement Witness, Gell-Mann Matrices, Linear Programming,

PACs Index: 03.65.Ud
\end{abstract} Feasible Region. 


\section{Introduction}

Entanglement is one of the interesting features of quantum systems. It is used as a physical resource in realization of many quantum information and quantum computation processes such as quantum cryptography, teleportation, dense coding and quantum key distribution [1, 2, 3] which cannot be realized in classical physics. So a strong motivation has been raised for the study of entanglement detection in an operational way. Though the fields of quantum information and quantum computation have been mainly built on the concept of qubits, however the exploration of qutrits has been attracted much attention in the recent years [4, 5, 6]. A qutrit, the simplest generalization of a qubit, is a system whose operators act on a three-dimensional Hilbert space. Qutrits have some interesting properties that account for their usefulness: they have improved the efficiency and security of many quantum information protocols, and it is expected that systems of entangled qutrits largely violate the non-locality via Bell tests [7]. Therefore, the detection of entanglement, that is, distinguishing between separable and entangled states, for two-qutrit systems is an important problem.

Among the different criteria to analyze the separability of quantum states, the entanglement witnesses (EWs) are of special interest since it has been proved that for any entangled state there exists at least one EW which detects its entanglement [8, 9]. The EWs are Hermitian operators which have non-negative expectation values over all separable states and detect some entangled states. Therefore, for construction of EWs, one needs to determine the minimum of this expectation value and to demand its non-negativity which lies in realm of the convex optimization problem. The linear programming (LP), as a special case of convex optimization, is one of the very useful approaches for constructing EWs [10, 11, 12, 13, 14, 15, 16] since it can be solved by using very efficient algorithms such as the simplex and interior-point methods ( see e.g. [17, 18]).

Inside the several problems concerning the EWs, the problem of how to construct EWs by a 
given set of operators has a great importance. In this paper, we use the Gell-Mann $\lambda$ matrices , a basis for the Lie algebra su(3) [19], to construct EWs for two-qutrit states. Two $\lambda$-diagonal and $\lambda$-non-diagonal cases are considered and it is shown that the problem of constructing such EWs can be reduced to a LP problem. We show that, as far as related to the LP method, the presented $\lambda$-diagonal EWs are all decomposable while a large number of $\lambda$-non-diagonal EWs are non-decomposable. To show the non-decomposability of these EWs, we find a class of bound entangled states or used known bound entangled states that can be detected by them. Also, it is noted that in determining the decomposability or non-decomposability of a given EW, the pure product states which have zero expectation values with it play a crucial role.

Determination of the feasible region is the main goal of any separability problem. So in the method of the present paper, it is tried to determine the feasible region exactly or approximately. When the feasible region was determined, any hyper-plane tangent to it would correspond to an optimal EW. In this way, we get all possible optimal EWs, including the pre-existent ones obtained by other methods.

The paper is organized as follows: In Section 2, we review the basic notions and definitions of EWs relevant to our study and describe a general approach of constructing EWs by the LP method. In Section 3, we consider the construction of $\lambda$-diagonal EWs and Section 4 is devoted to the construction of various $\lambda$-non-diagonal EWs. The paper is ended with a brief conclusion and three appendices.

\section{EWs and LP method}

\subsection{Entanglement witnesses}

First let us recall the definition of entanglement and separability. A bipartite quantum mixed state $\rho \in \mathcal{B}(\mathcal{H})$ (the Hilbert space of bounded operators acting on $\mathcal{H}=\mathcal{H}_{d_{1}} \otimes \mathcal{H}_{d_{2}}$ ) is called 
separable if it can be written as a convex combination of pure product states, that is

$$
\rho=\sum_{i} p_{i}\left|\alpha_{i}^{(1)}\right\rangle\left\langle\alpha_{i}^{(1)}|\otimes| \alpha_{i}^{(2)}\right\rangle\left\langle\alpha_{i}^{(2)}\right|
$$

where $\left|\alpha_{i}^{(j)}\right\rangle$ are arbitrary but normalized vectors lying in the $\mathcal{H}_{d_{j}}$, and $p_{i} \geq 0$ with $\sum_{i} p_{i}=1$. When this is not the case, $\rho$ is called entangled.

An entanglement witness $W$ is a Hermitian operator such that $\operatorname{Tr}\left(W \rho_{s}\right) \geq 0$ for all separable states $\rho_{s}$ and there exists at least one entangled state $\rho_{e}$ which can be detected by $W$, that is $\operatorname{Tr}\left(W \rho_{e}\right)<0$. The existence of an EW for any entangled state is a direct consequence of Hahn-Banach theorem [20] and the fact that the space of separable density operators is convex and closed.

Based on the notion of partial transpose, the EWs are classified into two classes: decomposable (d-EW) and non-decomposable (nd-EW). An EW $W$ is called decomposable if there exist positive operators $\mathcal{P}, \mathcal{Q}$ such that

$$
W=\mathcal{P}+\mathcal{Q}^{T_{1}}
$$

where $T_{1}$ denotes the partial transpose with respect to partite 1 and it is non-decomposable if it can not be written in this form [10]. Clearly a d-EW can not detect bound entangled states (entangled states with positive partial transpose (PPT) ) whereas there are some bound entangled states which can be detected by an nd-EW.

Usually one is interested in finding EWs $W$ which detect entangled states in an optimal way in the sense that when we subtract any positive operator from $W$ then it does not remain an EW anymore [21]. In other words, if there exist $\epsilon>0$ and a positive operator $\mathcal{P}$ such that $W^{\prime}=W-\epsilon \mathcal{P}$ is again an EW then we conclude that $W$ is not optimal, otherwise it is.

\subsection{Linear programming method}

This subsection is devoted to describing linear programming (LP) and a general approach for constructing EWs by the LP method exactly or approximately [17]. 
Let us consider a non-positive Hermitian operator of the form

$$
W=a_{0} I+\sum_{i} a_{i} Q_{i}
$$

where $Q_{i}$ are Hermitian operators and $a_{i}$ 's are real parameters with $a_{0}>0$. In this work, the $Q_{i}$ will be considered as tesor products of Gell-Mann matrices for Lie algebra su(3). We will attempt to choose the real parameters $a_{i}$ such that $W$ becomes an EW. To this aim, we introduce the maps

$$
P_{i}=\operatorname{Tr}\left(Q_{i} \rho_{s}\right)
$$

for any separable state $\rho_{s}$. The maps $P_{i}$ map the convex set of separable states into a bounded convex region which will be named feasible region. The first property of an EW is that its expectation value over any separable state is non-negative, i.e., the condition

$$
F_{W}:=\operatorname{Tr}\left(W \rho_{s}\right)=a_{0}+\sum_{i} a_{i} P_{i} \geq 0
$$

is satisfied for any point $\left(P_{1}, P_{2}, \ldots\right)$ of the feasible region. In order to satisfy this condition, it is sufficient that the minimum value of $F_{W}$ be non-negative. We note that the quantity $F_{W}$ achieves its minimum value for pure product states, since every separable mixed state $\rho_{s}$ can be written as a convex combination of pure product states, say $\rho_{s}=\sum_{i} p_{i}\left|\gamma_{i}\right\rangle\left\langle\gamma_{i}\right|$ with $p_{i} \geq 0$ and $\sum_{i} p_{i}=1$, whence we have

$$
\begin{gathered}
\operatorname{Tr}\left(W \rho_{s}\right)=\sum_{i} p_{i} \operatorname{Tr}\left(W\left|\gamma_{i}\right\rangle\left\langle\gamma_{i}\right|\right) \geq C_{\text {min }}, \\
\text { with } \quad C_{\text {min }}=\min _{|\gamma\rangle \in D_{\text {prod }}} \operatorname{Tr}(W|\gamma\rangle\langle\gamma|)
\end{gathered}
$$

where $D_{\text {prod }}$ denotes the set of pure product states. Here in this work we are interested in the EWs that their feasible regions are of simplex (or at most convex polygon) types. The manipulation of these EWs amounts to

$$
\begin{gathered}
\operatorname{minimize} \quad F_{W}=a_{0}+\sum_{i} a_{i} P_{i} \\
\text { subject to } \quad \sum_{i}\left(c_{i j} P_{i}-d_{j}\right) \geq 0 \quad j=1,2, \ldots
\end{gathered}
$$


where $c_{i j}$ and $d_{j}$ are parameters of hyper-planes surrounding the feasible regions. So the problem reduces to a LP problem. On the basis of the LP method, minimum of an objective function $F_{W}$ always occurs at the vertices of bounded feasible region. Therefore the vertices of the feasible region come from pure product states.

It is necessary to distinguish between two cases: (a) exactly soluble, and (b) approximately soluble EWs. In the case a the boundaries (constraints on $P_{i}$ ) come from the finite set of vertices arising from pure product states and construct a convex polygon, while in the case b the boundaries may not be hyper-planes. In this case we approximate the boundaries with hyper-planes and clearly some vertices of the approximated feasible region do not arise from pure product states. Both cases can be solved by the well-known simplex method. The simplex algorithm is a common algorithm used to solve an optimization problem with a polytope feasible region, such as a linear programming problem. Here, considering the scope of this paper, a complete treatment of the simplex algorithm is unnecessary; for a more complete treatment please refer to any LP text such as [17, 18].

\section{$3 \lambda$-diagonal EWs}

\subsection{Exactly soluble EWs}

In this subsection we consider $\lambda$-diagonal EWs which can be solved exactly by the LP method. Let us consider a situation that the Hermitian operator is as follows

$$
W=a_{0} I_{3} \otimes I_{3}+\sum_{k=1}^{8} a_{k} \lambda_{k} \otimes \lambda_{k}
$$

where $\lambda_{k}$ 's are the Gell-Mann matrices for Lie algebra su(3). Evidently when all of its nine eigenvalues are positive the above operator is positive; otherwise it may be an EW.

Now we attempt to choose the real parameters $a_{i}$ such that $W$ becomes an EW. To this aim, we need to minimize the expectation value of $W$ over all separable states and to demand its 
non-negativity. To reduce the problem to a LP problem and to determine the feasible region, we require to know the vertices, namely the extreme points, of the feasible region. Vertices of the feasible region come from pure product states and are listed in the table 1 where $\left|\lambda_{k} ; m\right\rangle$ is

\begin{tabular}{cccccccc|c}
\hline$P_{1}$ & $P_{2}$ & $P_{3}$ & $P_{4}$ & $P_{5}$ & $P_{6}$ & $P_{7}$ & $P_{8}$ & Product state \\
\hline \pm 1 & 0 & 0 & 0 & 0 & 0 & 0 & $\frac{1}{3}$ & $\left|\lambda_{1} ;+1\right\rangle \otimes\left|\lambda_{1} ; \pm 1\right\rangle$ \\
0 & \pm 1 & 0 & 0 & 0 & 0 & 0 & $\frac{1}{3}$ & $\left|\lambda_{2} ;+1\right\rangle \otimes\left|\lambda_{2} ; \pm 1\right\rangle$ \\
0 & 0 & \pm 1 & 0 & 0 & 0 & 0 & $\frac{1}{3}$ & $\left|\lambda_{3} ;+1\right\rangle \otimes\left|\lambda_{3} ; \pm 1\right\rangle$ \\
0 & 0 & $\frac{1}{4}$ & \pm 1 & 0 & 0 & 0 & $\frac{1}{12}$ & $\left|\lambda_{4} ;+1\right\rangle \otimes\left|\lambda_{4} ; \pm 1\right\rangle$ \\
0 & 0 & $\frac{1}{4}$ & 0 & \pm 1 & 0 & 0 & $\frac{1}{12}$ & $\left|\lambda_{5} ;+1\right\rangle \otimes\left|\lambda_{5} ; \pm 1\right\rangle$ \\
0 & 0 & $\frac{1}{4}$ & 0 & 0 & \pm 1 & 0 & $\frac{1}{12}$ & $\left|\lambda_{6} ;+1\right\rangle \otimes\left|\lambda_{6} ; \pm 1\right\rangle$ \\
0 & 0 & $\frac{1}{4}$ & 0 & 0 & 0 & \pm 1 & $\frac{1}{12}$ & $\left|\lambda_{7} ;+1\right\rangle \otimes\left|\lambda_{7} ; \pm 1\right\rangle$ \\
0 & 0 & 0 & 0 & 0 & 0 & 0 & $\frac{4}{3}$ & $\left|\lambda_{8} ; \frac{-2}{\sqrt{3}}\right\rangle \otimes\left|\lambda_{8} ; \frac{-2}{\sqrt{3}}\right\rangle$ \\
0 & 0 & 0 & 0 & 0 & 0 & 0 & $-\frac{2}{3}$ & $\left|\lambda_{8} ; \frac{1}{\sqrt{3}}\right\rangle \otimes\left|\lambda_{8} ; \frac{-2}{\sqrt{3}}\right\rangle$ \\
\hline
\end{tabular}

Table 1: The product vectors and coordinates of vertices for $\mathrm{W}$.

the eigenvector of $\lambda_{k}$ with eigenvalue $m$ and $P_{k}=\operatorname{Tr}\left(\lambda_{k} \otimes \lambda_{k}|\gamma\rangle\langle\gamma|\right)$ in which $|\gamma\rangle$ is an arbitrary pure product state.

The hyper-planes passing through the two vertices $\left(0,0,1,0,0,0,0, \frac{1}{3}\right)$ and $\left(0,0,0,0,0,0,0, \frac{4}{3}\right)$ together with other vertices except the vertex $\left(0,0,0,0,0,0,0,-\frac{2}{3}\right)$ have the form

$$
\frac{3}{4} \sum_{j=1}^{8}(-1)^{i_{j}} P_{j}-1=0 \quad ; \quad i_{3}=i_{8}=0 \quad \text { and } \quad i_{j} \in\{0,1\} \quad \text { for } \quad j \neq 3,8 .
$$

To locate the position of the feasible region with respect to these hyper-planes, we note that the origin $\left(P_{1}, \ldots, P_{8}\right)=(0, \ldots, 0)$ come from the pure product state

$$
\frac{1}{\sqrt{3}}\left(\begin{array}{c}
1 \\
1 \\
1
\end{array}\right) \otimes\left(\begin{array}{l}
0 \\
0 \\
1
\end{array}\right)
$$


and hence lies within the feasible region. On the other hand, for the origin we have

$$
\frac{3}{4} \sum_{j=1}^{8}(-1)^{i_{j}} P_{j}-1=-1<0 .
$$

Hence, the feasible region lies in one side of the hyper-planes (3.8). To make sure, it is proved that there exists no pure product state on the other side of these hyper-planes (for a proof, see appendix II). So the hyper-planes (3.8) are boundaries of the feasible region.

Each hyper-plane (3.8) corresponds to a positive operator or an EW. To see this, note that each hyper-plane (3.8) can be rewritten as

$$
\operatorname{Tr}\left(\left[I_{3} \otimes I_{3}-\frac{3}{4} \sum_{j=1}^{8}(-1)^{i_{j}} \lambda_{j} \otimes \lambda_{j}\right]|\gamma\rangle\langle\gamma|\right)=0
$$

with $|\gamma\rangle$ a pure product state that corresponds to a vertex of the feasible region. Hence, the operator

$$
I_{3} \otimes I_{3}-\frac{3}{4} \sum_{j=1}^{8}(-1)^{i} \lambda_{j} \otimes \lambda_{j}
$$

has positive expectation value over any pure product state and we conclude that it is a positive operator or an EW. To simplify the analysis, we consider the following cases with this in mind that in all cases $i_{3}=i_{8}=0$.

a- $i_{1}=i_{2} \quad, \quad i_{4}=i_{5} \quad, \quad i_{6}=i_{7}$.

This case contains eight operators which are all positive.

b- $i_{1} \neq i_{2} \quad, \quad i_{4} \neq i_{5} \quad, \quad i_{6} \neq i_{7}$.

The eight operators of this case are all d-EWs since they have some negative eigenvalues and their partial transposes with respect to the first partite which is equivalent to $\lambda_{i} \rightarrow-\lambda_{i}$ for $i=2,5,7$, yield one of the eight positive operators of case $\mathbf{a}$.

c- $i_{1}=i_{2}, i_{4} \neq i_{5}, i_{6} \neq i_{7} \quad ; \quad i_{1} \neq i_{2}, i_{4}=i_{5}, i_{6} \neq i_{7} \quad ; \quad i_{1} \neq i_{2}, i_{4} \neq i_{5}, i_{6}=i_{7}$.

This case contains 24 operators which are all d-EWs. For instance, consider the following one

$$
I_{3} \otimes I_{3}-\frac{3}{4}\left(\lambda_{1} \otimes \lambda_{1}+\lambda_{2} \otimes \lambda_{2}+\lambda_{3} \otimes \lambda_{3}+\lambda_{4} \otimes \lambda_{4}-\lambda_{5} \otimes \lambda_{5}+\lambda_{6} \otimes \lambda_{6}-\lambda_{7} \otimes \lambda_{7}+\lambda_{8} \otimes \lambda_{8}\right) .
$$


It can be written as

$$
\mathcal{P}+\mathcal{Q}^{T_{1}}
$$

in which the positive operators $\mathcal{P}$ and $\mathcal{Q}$ are

$$
\mathcal{P}=3\left|\psi_{1}\right\rangle\left\langle\psi_{1}\right| \quad, \quad \mathcal{Q}=3\left(\left|\psi_{2}\right\rangle\left\langle\psi_{2}|+| \psi_{3}\right\rangle\left\langle\psi_{3}\right|\right)
$$

Here $\left|\psi_{i}\right\rangle$ 's are states that to be defined in relation (II-6) of appendix III.

d- $i_{1} \neq i_{2}, i_{4}=i_{5}, i_{6}=i_{7} \quad ; \quad i_{1}=i_{2}, i_{4} \neq i_{5}, i_{6}=i_{7} \quad ; \quad i_{1}=i_{2}, i_{4}=i_{5}, i_{6} \neq i_{7}$.

The 24 operators of this case are all d-EWs since their partial transposes are equal to one of the $24 \mathrm{~d}-\mathrm{EW}$ s of case $\mathbf{c}$.

In summary, it was shown that among the 64 boundary hyper-planes (3.8) of the feasible region, eight of them correspond to positive operators and the others correspond to d-EWs. These 64 boundaries are hyper-planes by itself while as it is shown in the sequel, the remaining ones are not hyper-planes by itself and we have to approximate them by hyper-planes in order to apply the LP method.

The EW introduced in Eq. (74) of [6] for the isotropic qutrit states and also the EWs in Eqs. (118) and (134) of [22] are among the EWs of case $\mathbf{b}$.

\subsection{Approximately soluble EWs}

In this subsection we consider other boundaries of the feasible region for EWs of the form (3.7). We show that these are not hyper-planes by itself and we have to approximate them with hyper-planes.

At first, we choose two vertices $\left(0,0,1,0,0,0,0, \frac{1}{3}\right)$ and $\left(0,0,0,0,0,0,0,-\frac{2}{3}\right)$ together with

other vertices except the vertex $\left(0,0,0,0,0,0,0, \frac{4}{3}\right)$. The hyper-planes passing through these vertices have the form

$$
\frac{3}{2}\left[(-1)^{i_{1}} P_{1}+(-1)^{i_{2}} P_{2}+P_{3}-P_{8}\right]+\frac{3}{4}\left[(-1)^{i_{4}} P_{4}+(-1)^{i_{5}} P_{5}+(-1)^{i_{6}} P_{6}+(-1)^{i_{7}} P_{7}\right]-1=0
$$


where $i_{1}, i_{2}, i_{4}, \ldots, i_{7} \in\{0,1\}$. In appendix II, we find pure product states that maximizes the left hand side of (3.12) and show that this maximum is not zero but it is $\frac{3}{8}$. This implies that the hyper-planes (3.12) lie inside the feasible region and so are not really the boundaries of the feasible region. At first approximation, we approximate the corresponding real boundaries by hyper-planes that are parallel to the hyper-planes (3.12) and are tangent to real boundaries of the feasible region at pure product states giving the maximum value $\frac{3}{8}$. The consequence of the above approximation is that some vertices of the approximated feasible region do not come from pure product states. The EWs corresponding to these approximated boundary hyper-planes are as follows

$$
\begin{aligned}
W_{1}= & \frac{11}{8} I_{3} \otimes I_{3}-\frac{3}{2}\left[(-1)^{i_{1}} \lambda_{1} \otimes \lambda_{1}+(-1)^{i_{2}} \lambda_{2} \otimes \lambda_{2}+\lambda_{3} \otimes \lambda_{3}-\lambda_{8} \otimes \lambda_{8}\right] \\
& -\frac{3}{4}\left[(-1)^{i_{4}} \lambda_{4} \otimes \lambda_{4}+(-1)^{i_{5}} \lambda_{5} \otimes \lambda_{5}+(-1)^{i_{6}} \lambda_{6} \otimes \lambda_{6}+(-1)^{i_{7}} \lambda_{7} \otimes \lambda_{7}\right] .
\end{aligned}
$$

Among them we study the following one in detail

$W_{1}^{\prime}=\frac{11}{8} I_{3} \otimes I_{3}-\frac{3}{2}\left[\lambda_{1} \otimes \lambda_{1}+\lambda_{2} \otimes \lambda_{2}+\lambda_{3} \otimes \lambda_{3}-\lambda_{8} \otimes \lambda_{8}\right]-\frac{3}{4}\left[\lambda_{4} \otimes \lambda_{4}+\lambda_{5} \otimes \lambda_{5}+\lambda_{6} \otimes \lambda_{6}+\lambda_{7} \otimes \lambda_{7}\right]$.

To show the decomposability of $W_{1}^{\prime}$, we assume that it is decomposable and write

$$
W_{1}^{\prime}=\mathcal{P}+\mathcal{Q}^{T_{1}}
$$

Also, we note that $W_{1}^{\prime}$ vanishes on the pure product states $|\gamma\rangle=|\alpha\rangle \otimes|\beta\rangle$ in relation (I-4) of appendix II, i.e.,

$$
\begin{gathered}
\operatorname{Tr}\left(W_{1}^{\prime}|\gamma\rangle\langle\gamma|\right)=\left\langle\gamma\left|W_{1}^{\prime}\right| \gamma\right\rangle=\langle\gamma|\mathcal{P}| \gamma\rangle+\left\langle\gamma\left|\mathcal{Q}^{T_{1}}\right| \gamma\right\rangle \\
=\langle\gamma|\mathcal{P}| \gamma\rangle+\left\langle\alpha^{*}\left|\left\langle\beta|\mathcal{Q}| \alpha^{*}\right\rangle\right| \beta\right\rangle=0
\end{gathered}
$$

where $\left|\alpha^{*}\right\rangle$ is the complex conjugate of $|\alpha\rangle$. This requires that

$$
\langle\gamma|\mathcal{P}| \gamma\rangle=0 \quad \text { and } \quad\left\langle\alpha^{*}\left|\left\langle\beta|\mathcal{Q}| \alpha^{*}\right\rangle\right| \beta\right\rangle=0
$$

So the positive operators $\mathcal{P}$ and $\mathcal{Q}$ have to be orthogonal to all $|\gamma\rangle$ 's and $\left|\alpha^{*}\right\rangle \otimes|\beta\rangle$ 's resppectively. As it is shown in appendix II, such $\mathcal{P}$ and $\mathcal{Q}$ actually exist and hence $W_{1}^{\prime}$ is a d-EW. Similarly, we can show that EWs (3.13) are all d-EWs. 
In the same way, it can be shown that the remaining boundaries are also not hyper-planes by itself and the EWs corresponding to hyper-planes approximating them, i.e.,

$$
\begin{gathered}
W_{2}=2 I_{3} \otimes I_{3}-\frac{3}{2}\left[(-1)^{i_{1}} \lambda_{1} \otimes \lambda_{1}+(-1)^{i_{2}} \lambda_{2} \otimes \lambda_{2}-\lambda_{3} \otimes \lambda_{3}+(-1)^{i_{4}} \lambda_{4} \otimes \lambda_{4}\right. \\
\left.+(-1)^{i_{5}} \lambda_{5} \otimes \lambda_{5}+(-1)^{i_{6}} \lambda_{6} \otimes \lambda_{6}+(-1)^{i_{7}} \lambda_{7} \otimes \lambda_{7}-\lambda_{8} \otimes \lambda_{8}\right]
\end{gathered}
$$

and

$$
\begin{aligned}
W_{3} & =\frac{11}{8} I_{3} \otimes I_{3}-\frac{3}{4}\left[(-1)^{i_{1}} \lambda_{1} \otimes \lambda_{1}+(-1)^{i_{2}} \lambda_{2} \otimes \lambda_{2}-\lambda_{3} \otimes \lambda_{3}+\lambda_{8} \otimes \lambda_{8}\right] \\
& -\frac{9}{8}\left[(-1)^{i_{4}} \lambda_{4} \otimes \lambda_{4}+(-1)^{i_{5}} \lambda_{5} \otimes \lambda_{5}+(-1)^{i_{6}} \lambda_{6} \otimes \lambda_{6}+(-1)^{i_{7}} \lambda_{7} \otimes \lambda_{7}\right],
\end{aligned}
$$

are all d-EWs.

In summary, the EWs of the form (3.7) which correspond to boundaries or approximated boundaries hyper-planes of the feasible region, are all d-EWs. The other EWs of the form (3.7) can be written as a convex combination of a positive operator and one of the EWs coming from boundary hyper-planes [15]. So if all the boundaries would be hyper-planes by itself, we could say that $\lambda$-diagonal EWs are all decomposable. Therefore, it is tempting to add some off-diagonal terms $\lambda_{i} \otimes \lambda_{j}$ with $i \neq j$ to the operator of (3.7) in order to get nd-EWs which is the topic of the next section.

\section{$4 \lambda$-non-diagonal EWs}

\subsection{First category}

For the first ctegory of $\lambda$-non-diagonal EWs, let us consider a Hermitian operator of the form

$$
\begin{aligned}
\mathcal{W}= & a_{0} I_{3} \otimes I_{3}+\sum_{k=1}^{8} a_{k} \lambda_{k} \otimes \lambda_{k}+a_{1,2} \lambda_{1} \otimes \lambda_{2}+a_{2,1} \lambda_{2} \otimes \lambda_{1} \\
& +a_{4,5} \lambda_{4} \otimes \lambda_{5}+a_{5,4} \lambda_{5} \otimes \lambda_{4}+a_{6,7} \lambda_{6} \otimes \lambda_{7}+a_{7,6} \lambda_{7} \otimes \lambda_{6}
\end{aligned}
$$

Vertices of the feasible region coming from pure product states are listed in the table 2.

The hyper-plane passing through the two vertices

$$
\left(0,0,0,0,0,0,0, \frac{4}{3}, 0,0,0,0,0,0\right) \quad, \quad\left(0,0,1,0,0,0,0, \frac{1}{3}, 0,0,0,0,0,0\right)
$$




\begin{tabular}{cccccccccccccc|l}
\hline$P_{1}$ & $P_{2}$ & $P_{3}$ & $P_{4}$ & $P_{5}$ & $P_{6}$ & $P_{7}$ & $P_{8}$ & $P_{1,2}$ & $P_{2,1}$ & $P_{4,5}$ & $P_{5,4}$ & $P_{6,7}$ & $P_{7,6}$ & Product state \\
\hline \pm 1 & 0 & 0 & 0 & 0 & 0 & 0 & $\frac{1}{3}$ & 0 & 0 & 0 & 0 & 0 & 0 & $\left|\lambda_{1} ;+1\right\rangle\left|\lambda_{1} ; \pm 1\right\rangle$ \\
0 & \pm 1 & 0 & 0 & 0 & 0 & 0 & $\frac{1}{3}$ & 0 & 0 & 0 & 0 & 0 & 0 & $\left|\lambda_{2} ;+1\right\rangle\left|\lambda_{2} ; \pm 1\right\rangle$ \\
0 & 0 & \pm 1 & 0 & 0 & 0 & 0 & $\frac{1}{3}$ & 0 & 0 & 0 & 0 & 0 & 0 & $\left|\lambda_{3} ;+1\right\rangle\left|\lambda_{3} ; \pm 1\right\rangle$ \\
0 & 0 & 0 & 0 & 0 & 0 & 0 & $\frac{1}{3}$ & \pm 1 & 0 & 0 & 0 & 0 & 0 & $\left|\lambda_{1} ;+1\right\rangle\left|\lambda_{2} ; \pm 1\right\rangle$ \\
0 & 0 & 0 & 0 & 0 & 0 & 0 & $\frac{1}{3}$ & 0 & \pm 1 & 0 & 0 & 0 & 0 & $\left|\lambda_{2} ;+1\right\rangle\left|\lambda_{1} ; \pm 1\right\rangle$ \\
0 & 0 & $\frac{1}{4}$ & \pm 1 & 0 & 0 & 0 & $\frac{1}{12}$ & 0 & 0 & 0 & 0 & 0 & 0 & $\left|\lambda_{4} ;+1\right\rangle\left|\lambda_{4} ; \pm 1\right\rangle$ \\
0 & 0 & $\frac{1}{4}$ & 0 & \pm 1 & 0 & 0 & $\frac{1}{12}$ & 0 & 0 & 0 & 0 & 0 & 0 & $\left|\lambda_{5} ;+1\right\rangle\left|\lambda_{5} ; \pm 1\right\rangle$ \\
0 & 0 & $\frac{1}{4}$ & 0 & 0 & \pm 1 & 0 & $\frac{1}{12}$ & 0 & 0 & 0 & 0 & 0 & 0 & $\left|\lambda_{6} ;+1\right\rangle\left|\lambda_{6} ; \pm\right\rangle$ \\
0 & 0 & $\frac{1}{4}$ & 0 & 0 & 0 & \pm 1 & $\frac{1}{12}$ & 0 & 0 & 0 & 0 & 0 & 0 & $\left|\lambda_{7} ;+1\right\rangle\left|\lambda_{7} ; \pm\right\rangle$ \\
0 & 0 & $\frac{1}{4}$ & 0 & 0 & 0 & 0 & $\frac{1}{12}$ & 0 & 0 & \pm 1 & 0 & 0 & 0 & $\left|\lambda_{4} ;+1\right\rangle\left|\lambda_{5} ; \pm\right\rangle$ \\
0 & 0 & $\frac{1}{4}$ & 0 & 0 & 0 & 0 & $\frac{1}{12}$ & 0 & 0 & 0 & \pm 1 & 0 & 0 & $\left|\lambda_{5} ;+1\right\rangle\left|\lambda_{4} ; \pm\right\rangle$ \\
0 & 0 & $\frac{1}{4}$ & 0 & 0 & 0 & 0 & $\frac{1}{12}$ & 0 & 0 & 0 & 0 & \pm 1 & 0 & $\left|\lambda_{6} ;+1\right\rangle\left|\lambda_{7} ; \pm\right\rangle$ \\
0 & 0 & $\frac{1}{4}$ & 0 & 0 & 0 & 0 & $\frac{1}{12}$ & 0 & 0 & 0 & 0 & 0 & \pm 1 & $\left|\lambda_{7} ;+1\right\rangle\left|\lambda_{6} ; \pm\right\rangle$ \\
0 & 0 & 0 & 0 & 0 & 0 & 0 & $\frac{4}{3}$ & 0 & 0 & 0 & 0 & 0 & 0 & $\left|\lambda_{8} ; \frac{-2}{\sqrt{3}}\right\rangle\left|\lambda_{8} ; \frac{-2}{\sqrt{3}}\right\rangle$ \\
0 & 0 & 0 & 0 & 0 & 0 & 0 & $-\frac{2}{3}$ & 0 & 0 & 0 & 0 & 0 & 0 & $\left|\lambda_{8} ; \frac{1}{\sqrt{3}}\right\rangle\left|\lambda_{8} ; \frac{-2}{\sqrt{3}}\right\rangle$ \\
\hline
\end{tabular}

Table 2: The product vectors and coordinates of vertices for $\mathcal{W}$.

along with all other vertices having a coordinate +1 , is

$$
\frac{3}{4}\left(P_{1}+P_{2}+P_{1,2}+P_{2,1}+P_{3}+P_{4}+P_{5}+P_{4,5}+P_{5,4}+P_{6}+P_{7}+P_{6,7}+P_{7,6}+P_{8}\right)-1=0 .
$$

As the proof (see appendix II) shows, there exist pure product states that maximizes the left hand side of (4.19) and the maximum value is $\frac{3}{4}$, greater than zero. So this hyper-plane can not be a real boundary of the feasible region and we need to approximate the corresponding real boundary with the following hyper-plane parallel to it

$$
\frac{3}{4}\left(P_{1}+P_{2}+P_{1,2}+P_{2,1}+P_{3}+P_{4}+P_{5}+P_{4,5}+P_{5,4}+P_{6}+P_{7}+P_{6,7}+P_{7,6}+P_{8}\right)-\frac{7}{4}=0 .
$$


The corresponding EW of hyper-plane (4.20) is as follows

$$
\begin{gathered}
\mathcal{W}_{1}=\frac{7}{4} I_{3} \otimes I_{3}-\frac{3}{4}\left(\sum_{k=1}^{8} \lambda_{k} \otimes \lambda_{k}+\lambda_{1} \otimes \lambda_{2}+\lambda_{2} \otimes \lambda_{1}\right. \\
\left.+\lambda_{4} \otimes \lambda_{5}+\lambda_{5} \otimes \lambda_{4}+\lambda_{6} \otimes \lambda_{7}+\lambda_{7} \otimes \lambda_{6}\right)
\end{gathered}
$$

$\mathcal{W}_{1}$ is a nd-EW since it is able to detect a bound entangled state. To show this, we note that the density matrix

$$
\begin{gathered}
\rho=\frac{1}{9} I_{3} \otimes I_{3}+\frac{c}{6(a+2 c)}\left(\lambda_{1} \otimes \lambda_{1}+\lambda_{2} \otimes \lambda_{2}+\lambda_{1} \otimes \lambda_{2}+\lambda_{2} \otimes \lambda_{1}+\lambda_{4} \otimes \lambda_{4}+\lambda_{5} \otimes \lambda_{5}+\lambda_{4} \otimes \lambda_{5}\right. \\
\left.+\lambda_{5} \otimes \lambda_{4}+\lambda_{6} \otimes \lambda_{6}+\lambda_{7} \otimes \lambda_{7}+\lambda_{6} \otimes \lambda_{7}+\lambda_{7} \otimes \lambda_{6}\right)+\frac{a-c}{6(a+2 c)}\left(\lambda_{3} \otimes \lambda_{3}+\lambda_{8} \otimes \lambda_{8}\right)
\end{gathered}
$$

where $a$ and $c$ are non-negative real parameters such that $0 \leq c \leq \frac{a}{\sqrt{3}}$ is a PPT state. On the other hand,

$$
\operatorname{Tr}\left(\mathcal{W}_{1} \rho\right)=\frac{3}{4} \frac{a-2 c}{a+2 c} .
$$

It is seen that for $c>\frac{a}{2}$ the PPT state $\rho$ can be detected by $\mathcal{W}_{1}$. Hence, for $\frac{a}{2}<c \leq \frac{a}{\sqrt{3}}, \rho$ is a bound entangled state and $\mathcal{W}_{1}$ is a nd-EW.

This category contains $2^{14} \mathrm{EWs}$ corresponding to $2^{14}$ exact or approximated boundary hyper-planes of the feasible region. This set of EWs can be partitioned into 64 equivalence classes of size $2^{8}$ by the conjugation action of the exchange operator

$$
\Pi=\frac{1}{3} I_{3} \otimes I_{3}+\frac{1}{2} \sum_{k=1}^{8} \lambda_{k} \otimes \lambda_{k}
$$

which exchanges the two parties, and the operators $T \otimes I_{3}$, and $M_{i} \otimes I_{3}, i=1,2,3$ on it, where $\mathrm{T}$ is the transpose operator and

$$
M_{1}=\left(\begin{array}{ccc}
i & 0 & 0 \\
0 & 1 & 0 \\
0 & 0 & -1
\end{array}\right) \quad, \quad M_{2}=\left(\begin{array}{ccc}
1 & 0 & 0 \\
0 & i & 0 \\
0 & 0 & -1
\end{array}\right) \quad, \quad M_{3}=\left(\begin{array}{ccc}
1 & 0 & 0 \\
0 & -1 & 0 \\
0 & 0 & i
\end{array}\right) .
$$

Any two EWs are said to be equivalent if they can be transformed into each other by the above five operators or their possible products. The operators $M_{i}, i=1,2,3$ do not change GellMann matrices $\lambda_{3}$ and $\lambda_{8}$ while induce the following transformations on the other Gell-Mann 
matrices

$$
\begin{aligned}
& M_{1}: \quad \lambda_{1} \rightarrow-\lambda_{2}, \quad \lambda_{2} \rightarrow \lambda_{1}, \quad \lambda_{4} \rightarrow \lambda_{5}, \quad \lambda_{5} \rightarrow-\lambda_{4}, \quad \lambda_{6} \rightarrow-\lambda_{6}, \quad \lambda_{7} \rightarrow-\lambda_{7}, \\
& M_{2}: \quad \lambda_{1} \rightarrow \lambda_{2}, \quad \lambda_{2} \rightarrow-\lambda_{1}, \quad \lambda_{4} \rightarrow-\lambda_{4}, \quad \lambda_{5} \rightarrow-\lambda_{5}, \quad \lambda_{6} \rightarrow \lambda_{7}, \quad \lambda_{7} \rightarrow-\lambda_{6}, \\
& M_{3}: \quad \lambda_{1} \rightarrow-\lambda_{1}, \quad \lambda_{2} \rightarrow-\lambda_{2}, \quad \lambda_{4} \rightarrow \lambda_{5}, \quad \lambda_{5} \rightarrow-\lambda_{4}, \quad \lambda_{6} \rightarrow-\lambda_{7}, \quad \lambda_{7} \rightarrow \lambda_{6} .
\end{aligned}
$$

We have discussed one of the equivalence classes. As the discussion of the other classes is similar, we do not discuss them here.

\subsection{Second category}

For the second category of $\lambda$-non-diagonal EWs, let us consider the following Hermitian operator

$$
\mathcal{W}^{\prime}=a_{0} I_{3} \otimes I_{3}+\sum_{k=1}^{8} a_{k} \lambda_{k} \otimes \lambda_{k}+a_{3,8} \sqrt{3} \lambda_{3} \otimes \lambda_{8}+a_{8,3} \sqrt{3} \lambda_{8} \otimes \lambda_{3} .
$$

Vertices of the feasible region coming from pure product states are listed in the table 3 where $P_{3,8}=\operatorname{Tr}\left(\sqrt{3} \lambda_{3} \otimes \lambda_{8}|\gamma\rangle\langle\gamma|\right)$ and $P_{8,3}$ is defined similarly. To show the efficiency of the present method, we try to construct nd-EWs which are capable to detect the well-known one parameter family of bound entangled states

$$
\begin{gathered}
\rho_{b}=\frac{1}{9} I_{3} \otimes I_{3}+\frac{1}{21}\left(\lambda_{1} \otimes \lambda_{1}-\lambda_{2} \otimes \lambda_{2}+\lambda_{4} \otimes \lambda_{4}-\lambda_{5} \otimes \lambda_{5}+\lambda_{6} \otimes \lambda_{6}-\lambda_{7} \otimes \lambda_{7}\right) \\
-\frac{1}{84}\left(\lambda_{3} \otimes \lambda_{3}+\lambda_{8} \otimes \lambda_{8}\right)-\frac{\sqrt{3}}{84}(5-2 b)\left(\lambda_{3} \otimes \lambda_{8}-\lambda_{8} \otimes \lambda_{3}\right), \quad 0 \leq b \leq 5,
\end{gathered}
$$

introduced in [23]. The Horodecki states $\rho_{b}$ are PPT for $1 \leq b \leq 4$ and as it was shown in [23, 24], the states are separable for $2 \leq b \leq 3$ and bound entangled for $1 \leq b<2$ and $3<b \leq 4$. In [24], Doherty and et al. introduced an nd-EW which detects the Horodecki bound entangled states $\rho_{b}$ for $3<b \leq 4$.

Let us proceed to construct an nd-EW which detects $\rho_{b}$ for $3<b \leq 4$. To this aim, we 


\begin{tabular}{cccccccccc|l}
\hline$P_{1}$ & $P_{2}$ & $P_{3}$ & $P_{4}$ & $P_{5}$ & $P_{6}$ & $P_{7}$ & $P_{8}$ & $P_{3,8}$ & $P_{8,3}$ & Product state \\
\hline \pm 1 & 0 & 0 & 0 & 0 & 0 & 0 & $\frac{1}{3}$ & 0 & 0 & $\left|\lambda_{1} ;+1\right\rangle\left|\lambda_{1} ; \pm 1\right\rangle$ \\
0 & \pm 1 & 0 & 0 & 0 & 0 & 0 & $\frac{1}{3}$ & 0 & 0 & $\left|\lambda_{2} ;+1\right\rangle\left|\lambda_{2} ; \pm 1\right\rangle$ \\
0 & 0 & +1 & 0 & 0 & 0 & 0 & $\frac{1}{3}$ & \pm 1 & \pm 1 & $\left|\lambda_{3} ; \pm 1\right\rangle\left|\lambda_{3} ; \pm 1\right\rangle$ \\
0 & 0 & -1 & 0 & 0 & 0 & 0 & $\frac{1}{3}$ & \pm 1 & $\mp 1$ & $\left|\lambda_{3} ; \pm 1\right\rangle\left|\lambda_{3} ; \mp 1\right\rangle$ \\
0 & 0 & $\frac{1}{4}$ & \pm 1 & 0 & 0 & 0 & $\frac{1}{12}$ & $-\frac{1}{4}$ & $-\frac{1}{4}$ & $\left|\lambda_{4} ;+1\right\rangle\left|\lambda_{4} ; \pm 1\right\rangle$ \\
0 & 0 & $\frac{1}{4}$ & 0 & \pm 1 & 0 & 0 & $\frac{1}{12}$ & $-\frac{1}{4}$ & $-\frac{1}{4}$ & $\left|\lambda_{5} ;+1\right\rangle\left|\lambda_{5} ; \pm 1\right\rangle$ \\
0 & 0 & $\frac{1}{4}$ & 0 & 0 & \pm 1 & 0 & $\frac{1}{12}$ & $\frac{1}{4}$ & $\frac{1}{4}$ & $\left|\lambda_{6} ;+1\right\rangle\left|\lambda_{6} ; \pm\right\rangle$ \\
0 & 0 & $\frac{1}{4}$ & 0 & 0 & 0 & \pm 1 & $\frac{1}{12}$ & $\frac{1}{4}$ & $\frac{1}{4}$ & $\left|\lambda_{7} ;+1\right\rangle\left|\lambda_{7} ; \pm\right\rangle$ \\
0 & 0 & 0 & 0 & 0 & 0 & 0 & $\frac{4}{3}$ & 0 & 0 & $\left|\lambda_{8} ; \frac{-2}{\sqrt{3}}\right\rangle\left|\lambda_{8} ; \frac{-2}{\sqrt{3}}\right\rangle$ \\
0 & 0 & 0 & 0 & 0 & 0 & 0 & $-\frac{2}{3}$ & \pm 2 & 0 & $\left|\lambda_{8} ; \frac{1}{\sqrt{3}}\right\rangle\left|\lambda_{8} ; \frac{-2}{\sqrt{3}}\right\rangle$ \\
0 & 0 & 0 & 0 & 0 & 0 & 0 & $-\frac{2}{3}$ & 0 & \pm 2 & $\left|\lambda_{8} ; \frac{-2}{\sqrt{3}}\right\rangle\left|\lambda_{3} ; \frac{1}{\sqrt{3}}\right\rangle$ \\
\hline
\end{tabular}

Table 3: The product vectors and coordinates of vertices for $\mathcal{W}^{\prime}$.

begin with the following ten vertices of the feasible region

$$
\begin{array}{ccc}
\left(1,0,0,0,0,0,0, \frac{1}{3}, 0,0\right), & \left(0,-1,0,0,0,0,0, \frac{1}{3}, 0,0\right), & \left(0,0,-1,0,0,0,0, \frac{1}{3}, 1,-1\right), \\
\left(0,0, \frac{1}{4}, 1,0,0,0, \frac{1}{12},-\frac{1}{4},-\frac{1}{4}\right), & \left(0,0, \frac{1}{4}, 0,-1,0,0, \frac{1}{12},-\frac{1}{4},-\frac{1}{4}\right), & \left(0,0, \frac{1}{4}, 0,0,1,0, \frac{1}{12}, \frac{1}{4}, \frac{1}{4}\right), \\
\left(0,0, \frac{1}{4}, 0,0,0,-1, \frac{1}{12}, \frac{1}{4}, \frac{1}{4}\right), & \left(0,0,0,0,0,0,0,-\frac{2}{3}, 2,0\right), & \left(0,0,0,0,0,0,0,-\frac{2}{3}, 0,-2\right), \\
\left(0,0,0,0,0,0,0, \frac{4}{3}, 0,0\right) . &
\end{array}
$$

The hyper-plane passing through these vertices has the form

$$
\frac{3}{4}\left(P_{1}-P_{2}+P_{4}-P_{5}+P_{6}-P_{7}\right)+\frac{3}{4}\left(P_{3}+P_{8}\right)+\frac{3 \sqrt{3}}{4}\left(P_{3,8}-P_{8,3}\right)-1=0 .
$$

Some calculations show that the maximum value of the left hand side is $\frac{3}{8}$. The pure product state giving this maximum value lies on the boundary of the feasible region and we replace its corresponding point with one of the above ten vertices. Then we pass a hyper-plane through the second set of ten points and find pure product states maximizing it. Again, we replace the corresponding points of these states with suitable points of the second set. By continuing this process, we end up with ten vertices of the table 4. An analogous argument as above give the EW 


\begin{tabular}{|c|c|c|c|c|c|c|c|c|c|c|c|c|}
\hline$P_{1}$ & $P_{2}$ & $P_{3}$ & $P_{4}$ & $P_{5}$ & $P_{6}$ & $P_{7}$ & $P_{8}$ & $P_{3,8}$ & $P_{8,3}$ & \multicolumn{3}{|c|}{ Product state } \\
\hline 0 & 0 & -1 & 0 & 0 & 0 & 0 & $\frac{1}{3}$ & 1 & -1 & & 0 & $\otimes$ \\
\hline 0 & 0 & 0 & 0 & 0 & 0 & 0 & $-\frac{2}{3}$ & 2 & 0 & & 1 & $\otimes$ \\
\hline 0 & 0 & 0 & 0 & 0 & 0 & 0 & $-\frac{2}{3}$ & 0 & -2 & & 0 & $\otimes$ \\
\hline$\frac{4}{9}$ & 0 & 0 & $\frac{4}{9}$ & 0 & $\frac{4}{9}$ & 0 & 0 & 0 & 0 & $\frac{1}{\sqrt{3}}$ & 1 & $\otimes \frac{1}{\sqrt{3}}$ \\
\hline$\frac{4}{9}$ & 0 & 0 & $\frac{1}{9}$ & $-\frac{1}{3}$ & $\frac{1}{9}$ & $-\frac{1}{3}$ & 0 & 0 & 0 & $\frac{1}{1}$ & 1 & $\otimes \frac{1}{\sqrt{3}}$ \\
\hline 0 & $-\frac{4}{9}$ & 0 & 0 & $-\frac{4}{9}$ & $\frac{4}{9}$ & 0 & 0 & 0 & 0 & $\frac{1}{\sqrt{3}}$ & 1 & $\otimes \frac{1}{\sqrt{3}}$ \\
\hline 0 & $-\frac{4}{9}$ & 0 & $\frac{4}{9}$ & 0 & 0 & $-\frac{4}{9}$ & 0 & 0 & 0 & $\frac{1}{\sqrt{3}}$ & & $\otimes \frac{1}{\sqrt{3}}$ \\
\hline 0 & 0 & $\frac{3}{16}$ & 0 & 0 & $\frac{3}{4}$ & 0 & $-\frac{5}{48}$ & $\frac{15}{16}$ & $-\frac{1}{16}$ & $\frac{1}{2}$ & $\begin{array}{c}0 \\
\sqrt{3}\end{array}$ & $\otimes \frac{1}{2}$ \\
\hline$\frac{64}{225}$ & 0 & 0 & $\frac{112}{225}$ & 0 & $\frac{112}{225}$ & 0 & $\frac{4}{75}$ & 0 & 0 & $\frac{1}{\sqrt{15}}$ & $\begin{array}{l}2 \\
2\end{array}$ & $\otimes \frac{1}{\sqrt{15}}$ \\
\hline$\frac{2464}{9025}$ & 0 & $\frac{81}{1444}$ & $\frac{2592}{9025}$ & 0 & $\frac{6237}{9025}$ & 0 & $\frac{2809}{108300}$ & $\frac{477}{7220}$ & $\frac{477}{7220}$ & $\frac{1}{\sqrt{190}}$ & $\begin{array}{c}\sqrt{32} \\
\sqrt{77} \\
9\end{array}$ & $\otimes \frac{1}{\sqrt{190}}$ \\
\hline
\end{tabular}

Table 4: The product vectors and coordinates of vertices for $\mathcal{W}^{\prime}{ }_{1}$. Here $\omega=\exp \left(\frac{2 i \pi}{3}\right)$ and $i=\sqrt{-1}$.

$$
\begin{aligned}
\mathcal{W}_{1}^{\prime}=\frac{809}{790} I_{3} \otimes I_{3}-\frac{1}{6320} & {\left[2553\left(\lambda_{1} \otimes \lambda_{1}-\lambda_{2} \otimes \lambda_{2}\right)+5227\left(\lambda_{4} \otimes \lambda_{4}-\lambda_{5} \otimes \lambda_{5}\right)\right]-\frac{161}{158}\left(\lambda_{6} \otimes \lambda_{6}-\lambda_{7} \otimes \lambda_{7}\right) } \\
+ & \frac{501}{790}\left(\lambda_{3} \otimes \lambda_{3}+\lambda_{8} \otimes \lambda_{8}\right)-\frac{114 \sqrt{3}}{395}\left(\lambda_{3} \otimes \lambda_{8}-\lambda_{8} \otimes \lambda_{3}\right) .
\end{aligned}
$$

which corresponds to the hyper-plane tangent to the feasible region and parallel to the hyper-plane passing through the vertices of the table 4 . The nd-EW $\mathcal{W}^{\prime}{ }_{1}$ detects the Horodecki states $\rho_{b}$ for $\frac{2869}{912}(\simeq 3.146)<b \leq 4$. This nd-EW is very similar to the nd-EW introduced in [24].

To construct another nd-EW which detects $\rho_{b}$ for $1 \leq b<2$, we replace the first three points and the 8 th point of the table 4 with the three points

$$
\left(0,0,-1,0,0,0,0, \frac{1}{3},-1,1\right), \quad\left(0,0,0,0,0,0,0,-\frac{2}{3},-2,0\right), \quad\left(0,0,0,0,0,0,0,-\frac{2}{3}, 0,2\right),
$$


of the table 3 and the point $\left(0,0, \frac{3}{16}, 0,0, \frac{3}{4}, 0,-\frac{5}{48},-\frac{15}{16}, \frac{1}{16}\right)$ respectively. The last point corresponds to a pure product state coming from interchanging the states of two parties in the pure product state of the 8th point of the table 4 . The hyper-plane tangent to the feasible region and parallel to the hyper-plane passing through the new ten points, corresponds to the EW

$$
\begin{aligned}
\mathcal{W}_{2}^{\prime}=\frac{809}{790} I_{3} \otimes I_{3}-\frac{1}{6320} & {\left[2553\left(\lambda_{1} \otimes \lambda_{1}-\lambda_{2} \otimes \lambda_{2}\right)+5227\left(\lambda_{4} \otimes \lambda_{4}-\lambda_{5} \otimes \lambda_{5}\right)\right]-\frac{161}{158}\left(\lambda_{6} \otimes \lambda_{6}-\lambda_{7} \otimes \lambda_{7}\right) } \\
+ & \frac{501}{790}\left(\lambda_{3} \otimes \lambda_{3}+\lambda_{8} \otimes \lambda_{8}\right)+\frac{114 \sqrt{3}}{395}\left(\lambda_{3} \otimes \lambda_{8}-\lambda_{8} \otimes \lambda_{3}\right) .
\end{aligned}
$$

The nd-EW $\mathcal{W}^{\prime}{ }_{2}$ detects the Horodecki states $\rho_{b}$ for $1 \leq b<\frac{89}{48}(\simeq 1.854)$.

It is noted that the EW (4.29) can be obtained from EW (4.28) by the exchange operator $\Pi$ defined in (4.23), i.e.,

$$
\mathcal{W}_{2}^{\prime}=\Pi \mathcal{W}_{1}^{\prime} \Pi^{\dagger}
$$

In fact, this category contains $13 \times 2^{6}$ EWs corresponding to $5 \times 2^{6}$ exact or approximated boundary hyper-planes of the feasible region. This set of EWs can be partitioned into 36 equivalence classes, 20 classes of size 8 and 16 classes of size 16, by the conjugation action of exchange operator $\Pi$ and the operators $T \otimes I_{3}, M_{1}^{2} \otimes I_{3}$ and $M_{2}^{2} \otimes I_{3}$ on it, where $\mathrm{T}$ is the transpose operator and $M_{1}$ and $M_{2}$ are operators defined in (4.24). Any two EWs are said to be equivalent if they can be transformed into each other by the above four operators or their possible products. So we have studied one of the equivalence classes of size 16 which contains the two EWs $\mathcal{W}_{1}^{\prime}$ and $\mathcal{W}^{\prime}{ }_{2}$. The other classes can be studied similarly.

\section{Conclusion}

We have considered the EWs that can be constructed from Gell-Mann matrices for Lie algebra su(3) by using the Linear programming method. The general form of $\lambda$-diagonal and two cases of $\lambda$-nondiagonal EWs have been discussed in detail. It has been shown that in all considered cases the feasible region is a polygon approximately. In $\lambda$-diagonal case, the boundaries of the polygon correspond to d-EWs or positive operators which in turn implies that up to our approximation, all $\lambda$-diagonal EWs are decomposable. However, in $\lambda$-non-diagonal case, a large number of boundaries of the polygon 
correspond to nd-EWs. The decomposability of presented $\lambda$-diagonal EWs has been proved by writing them explicitly in decomposable form or by using the pure product states having zero expectation values with them. To show the non-decomposability of some EWs in $\lambda$-non-diagonal case, we have constructed a class of bound entangled states or used known bound entangled states that can be detected by them. Although the method may be tedious but as it was shown in the case of Horodecki bound entangled states, it clarify that in principle one can construct suitable $\lambda$-non-diagonal EWs for any two-qutrit bound entangled state.

Since the boundaries of the feasible region in $\lambda$-diagonal EWs are not all hyper-planes by itself, it remains as an open problem to show that $\lambda$-diagonal EWs are all decomposable. 


\section{Appendix I:}

\section{Lie algebra $\mathrm{su}(3)$}

The real Lie algebra $\mathrm{su}(3)$ is the algebra of the anti-Hermitian $3 \times 3$ matrices with vanishing trace. The standard Hermitian basis of $\mathrm{su}(3)$ consists of the Gell-Mann $\lambda$ matrices:

$$
\begin{aligned}
& \lambda_{1}=\left(\begin{array}{ccc}
0 & 1 & 0 \\
1 & 0 & 0 \\
0 & 0 & 0
\end{array}\right) \quad \lambda_{2}=\left(\begin{array}{ccc}
0 & -i & 0 \\
i & 0 & 0 \\
0 & 0 & 0
\end{array}\right) \quad \lambda_{3}=\left(\begin{array}{ccc}
1 & 0 & 0 \\
0 & -1 & 0 \\
0 & 0 & 0
\end{array}\right) \\
& \lambda_{4}=\left(\begin{array}{ccc}
0 & 0 & 1 \\
0 & 0 & 0 \\
1 & 0 & 0
\end{array}\right) \quad \lambda_{5}=\left(\begin{array}{ccc}
0 & 0 & -i \\
0 & 0 & 0 \\
i & 0 & 0
\end{array}\right) \quad \lambda_{6}=\left(\begin{array}{ccc}
0 & 0 & 0 \\
0 & 0 & 1 \\
0 & 1 & 0
\end{array}\right) \\
& \lambda_{7}=\left(\begin{array}{ccc}
0 & 0 & 0 \\
0 & 0 & -i \\
0 & i & 0
\end{array}\right) \quad \lambda_{8}=\frac{1}{\sqrt{3}}\left(\begin{array}{ccc}
1 & 0 & 0 \\
0 & 1 & 0 \\
0 & 0 & -2
\end{array}\right)
\end{aligned}
$$

which fulfill the condition

$$
\operatorname{Tr}\left(\lambda_{i} \lambda_{j}\right)=2 \delta_{i j}
$$

and the squares of expectation values of $\lambda_{i}$ 's over an arbitrary vector $|\alpha\rangle$ sum to $\frac{4}{3}$; i.e.,

$$
\sum_{i=1}^{8}\left(\left\langle\alpha\left|\lambda_{i}\right| \alpha\right\rangle\right)^{2}=\frac{4}{3}
$$

Here, for completeness, we give a proof for the above equality. Let $|\alpha\rangle=\alpha_{0}|0\rangle+\alpha_{1}|1\rangle+\alpha_{2}|2\rangle$ be an arbitrary state. Then we can write

$$
\begin{array}{lll}
\left\langle\alpha\left|\lambda_{1}\right| \alpha\right\rangle=2 \operatorname{Re}\left(\alpha_{0}^{*} \alpha_{1}\right) \quad, \quad & \left\langle\alpha\left|\lambda_{2}\right| \alpha\right\rangle=2 \operatorname{Im}\left(\alpha_{0}^{*} \alpha_{1}\right) \\
\left\langle\alpha\left|\lambda_{3}\right| \alpha\right\rangle=\left|\alpha_{0}\right|^{2}-\left|\alpha_{1}\right|^{2} \quad, & \left\langle\alpha\left|\lambda_{4}\right| \alpha\right\rangle=2 \operatorname{Re}\left(\alpha_{0}^{*} \alpha_{2}\right) \\
\left\langle\alpha\left|\lambda_{5}\right| \alpha\right\rangle=2 \operatorname{Im}\left(\alpha_{0}^{*} \alpha_{2}\right) \quad, & \left\langle\alpha\left|\lambda_{6}\right| \alpha\right\rangle=2 \operatorname{Re}\left(\alpha_{1}^{*} \alpha_{2}\right) \\
\left\langle\alpha\left|\lambda_{7}\right| \alpha\right\rangle=2 \operatorname{Im}\left(\alpha_{1}^{*} \alpha_{2}\right) \quad, & \left\langle\alpha\left|\lambda_{8}\right| \alpha\right\rangle=\frac{1}{\sqrt{3}}-\frac{3}{\sqrt{3}}\left|\alpha_{2}\right|^{2}
\end{array}
$$

whence

$$
\sum_{i=1}^{8}\left(\left\langle\alpha\left|\lambda_{i}\right| \alpha\right\rangle\right)^{2}=4\left|\alpha_{0}\right|^{2}\left|\alpha_{1}\right|^{2}+\left(\left|\alpha_{0}\right|^{2}-\left|\alpha_{1}\right|^{2}\right)^{2}+4\left|\alpha_{0}\right|^{2}\left|\alpha_{2}\right|^{2}+4\left|\alpha_{1}\right|^{2}\left|\alpha_{2}\right|^{2}+\frac{1}{3}\left(1-3\left|\alpha_{2}\right|^{2}\right)^{2}
$$




$$
\begin{aligned}
& =\left(\left|\alpha_{0}\right|^{2}+\left|\alpha_{1}\right|^{2}\right)^{2}+4\left|\alpha_{2}\right|^{2}\left(\left|\alpha_{0}\right|^{2}+\left|\alpha_{1}\right|^{2}\right)+\frac{1}{3}\left(1-3\left|\alpha_{2}\right|^{2}\right)^{2} \\
& =\left(\left|\alpha_{0}\right|^{2}+\left|\alpha_{1}\right|^{2}\right)\left(\left|\alpha_{0}\right|^{2}+\left|\alpha_{1}\right|^{2}+4\left|\alpha_{2}\right|^{2}\right)+\frac{1}{3}\left(1-3\left|\alpha_{2}\right|^{2}\right)^{2}
\end{aligned}
$$

noting the normalization condition

$$
\left|\alpha_{0}\right|^{2}+\left|\alpha_{1}\right|^{2}+\left|\alpha_{2}\right|^{2}=1
$$

the above statement can be written as

$$
\left(1-\left|\alpha_{2}\right|^{2}\right)\left(1+3\left|\alpha_{2}\right|^{2}\right)+\frac{1}{3}\left(1-3\left|\alpha_{2}\right|^{2}\right)^{2}=\frac{4}{3}
$$

\section{Appendix II:}

\section{Proving the equalities:}

In this appendix we give the proof of equalities for some boundary hyper-planes.

\section{The proof of (3.8):}

Since the proofs are similar, we give a proof only for the following hyper-plane

$$
\frac{3}{4} \sum_{j=1}^{8} P_{j}-1=0
$$

Let $|\gamma\rangle=|\alpha\rangle \otimes|\beta\rangle$ be an arbitrary pure product state. Then, by definition, we have

$$
\frac{3}{4} \sum_{j=1}^{8} P_{j}=\frac{3}{4} \sum_{j=1}^{8}\left\langle\alpha\left|\lambda_{j}\right| \alpha\right\rangle\left\langle\beta\left|\lambda_{j}\right| \beta\right\rangle
$$

Using the Cauchy-Schwartz inequality and (I-1), we get the result

$$
\leq \frac{3}{4}\left(\sum_{j=1}^{8}\left(\left\langle\alpha\left|\lambda_{j}\right| \alpha\right\rangle\right)^{2}\right)^{\frac{1}{2}}\left(\sum_{j=1}^{8}\left(\left\langle\beta\left|\lambda_{j}\right| \beta\right\rangle\right)^{2}\right)^{\frac{1}{2}}=\frac{3}{4} \sqrt{\frac{4}{3}} \sqrt{\frac{4}{3}}=1 .
$$

\section{The proof of (3.12):}

Since the proofs are similar, we give the proof only for the following one

$$
\frac{3}{2}\left[P_{1}+P_{2}+P_{3}-P_{8}\right]+\frac{3}{4}\left[P_{4}+P_{5}+P_{6}+P_{7}\right]-1=0
$$

By definition of $P_{i}$ 's and by noting to $(\overline{\mathrm{I}-2})$, the lhs of this equality can be written as

$$
\text { lhs }=\frac{3}{2}\left[4 \operatorname{Re}\left(\alpha_{0}^{*} \alpha_{1}\right) \operatorname{Re}\left(\beta_{0}^{*} \beta_{1}\right)+4 \operatorname{Im}\left(\alpha_{0}^{*} \alpha_{1}\right) \operatorname{Im}\left(\beta_{0}^{*} \beta_{1}\right)+\left(\left|\alpha_{0}\right|^{2}-\left|\alpha_{1}\right|^{2}\right)\left(\left|\beta_{0}\right|^{2}-\left|\beta_{1}\right|^{2}\right)\right.
$$




$$
\begin{gathered}
\left.-\frac{1}{3}\left(1-3\left|\alpha_{2}\right|^{2}\right)\left(1-3\left|\beta_{2}\right|^{2}\right)\right]+\frac{3}{4}\left[4 \operatorname{Re}\left(\alpha_{0}^{*} \alpha_{2}\right) \operatorname{Re}\left(\beta_{0}^{*} \beta_{2}\right) 4 \operatorname{Im}\left(\alpha_{0}^{*} \alpha_{2}\right) \operatorname{Im}\left(\beta_{0}^{*} \beta_{2}\right)\right. \\
\left.+4 \operatorname{Re}\left(\alpha_{1}^{*} \alpha_{2}\right) \operatorname{Re}\left(\beta_{1}^{*} \beta_{2}\right)+4 \operatorname{Im}\left(\alpha_{1}^{*} \alpha_{2}\right) \operatorname{Im}\left(\beta_{1}^{*} \beta_{2}\right)\right]-1 \\
=-1+6\left|\alpha_{0}\right|\left|\alpha_{1}\right|\left|\beta_{0}\right|\left|\beta_{1}\right| \cos \left(\theta_{01}-\theta_{01}^{\prime}\right)+\frac{3}{2}\left(\left|\alpha_{0}\right|^{2}-\left|\alpha_{1}\right|^{2}\right)\left(\left|\beta_{0}\right|^{2}-\left|\beta_{1}\right|^{2}\right) \\
-\frac{1}{2}\left(1-3\left|\alpha_{2}\right|^{2}\right)\left(1-3\left|\beta_{2}\right|^{2}\right)+3\left|\alpha_{0}\right|\left|\alpha_{2}\right|\left|\beta_{0}\right|\left|\beta_{2}\right| \cos \left(\theta_{02}-\theta_{02}^{\prime}\right)+3\left|\alpha_{1}\right|\left|\alpha_{2}\right|\left|\beta_{1}\right|\left|\beta_{2}\right| \cos \left(\theta_{12}-\theta_{12}^{\prime}\right) \\
\leq-1+6\left|\alpha_{0}\right|\left|\alpha_{1}\right|\left|\beta_{0}\right|\left|\beta_{1}\right|+\frac{3}{2}\left(\left|\alpha_{0}\right|^{2}-\left|\alpha_{1}\right|^{2}\right)\left(\left|\beta_{0}\right|^{2}-\left|\beta_{1}\right|^{2}\right) \\
-\frac{1}{2}\left(1-3\left|\alpha_{2}\right|^{2}\right)\left(1-3\left|\beta_{2}\right|^{2}\right)+3\left|\alpha_{0}\right|\left|\alpha_{2}\right|\left|\beta_{0}\right|\left|\beta_{2}\right|+3\left|\alpha_{1}\right|\left|\alpha_{2}\right|\left|\beta_{1}\right|\left|\beta_{2}\right| \\
=-1+3\left|\alpha_{2}\right|\left|\beta_{2}\right|\left(\left|\alpha_{0}\right|\left|\beta_{0}\right|+\left|\alpha_{1}\right|\left|\beta_{1}\right|+\left|\alpha_{2}\right|\left|\beta_{2}\right|\right)+\frac{3}{2}\left(\left|\alpha_{0}\right|\left|\beta_{0}\right|+\left|\alpha_{1}\right|\left|\beta_{1}\right|\right)^{2}-\frac{3}{2}\left(\left|\alpha_{0}\right|\left|\beta_{1}\right|-\left|\alpha_{1}\right|\left|\beta_{0}\right|\right)^{2} \\
+\frac{3}{2}\left(\left|\alpha_{2}\right|^{2}+\left|\beta_{2}\right|^{2}-5\left|\alpha_{2}\right|^{2}\left|\beta_{2}\right|^{2}-\frac{1}{3}\right)
\end{gathered}
$$

By using the Cauchy-Schwartz inequality, we have

$$
\leq-1+3\left|\alpha_{2}\right|\left|\beta_{2}\right|+\frac{3}{2}\left[\left(\left|\alpha_{0}\right|\left|\beta_{0}\right|+\left|\alpha_{1}\right|\left|\beta_{1}\right|\right)^{2}-\left(\left|\alpha_{0}\right|\left|\beta_{1}\right|-\left|\alpha_{1}\right|\left|\beta_{0}\right|\right)^{2}+\left|\alpha_{2}\right|^{2}+\left|\beta_{2}\right|^{2}-5\left|\alpha_{2}\right|^{2}\left|\beta_{2}\right|^{2}-\frac{1}{3}\right]
$$

By the following parametrization

$$
\begin{array}{ll}
\left|\alpha_{0}\right|=\sin \theta \cos \varphi & \left|\beta_{0}\right|=\sin \theta^{\prime} \cos \varphi^{\prime} \\
\left|\alpha_{1}\right|=\sin \theta \sin \varphi & \left|\beta_{1}\right|=\sin \theta^{\prime} \sin \varphi^{\prime} \\
\left|\alpha_{2}\right|=\cos \theta & \left|\beta_{2}\right|=\cos \theta^{\prime}
\end{array}
$$

where $\theta, \theta^{\prime}, \varphi, \varphi^{\prime} \in\left[0, \frac{\pi}{2}\right]$, the above statement can be written as

$$
3 \cos \theta \cos \theta^{\prime}+\frac{3}{2}\left[\sin ^{2} \theta \sin ^{2} \theta^{\prime} \cos 2\left(\varphi-\varphi^{\prime}\right)+\cos ^{2} \theta+\cos ^{2} \theta^{\prime}-5 \cos ^{2} \theta \cos ^{2} \theta^{\prime}-\frac{1}{3}\right]-1
$$

Since the Cauchy-Schwartz inequality becomes an equality when the two vectors are parallel, we must have $\theta=\theta^{\prime}$ and $\varphi=\varphi^{\prime}$. Then

$$
\text { lhs } \leq 3 \cos ^{2} \theta-6 \cos ^{4} \theta
$$

The lhs attains the maximum value $\frac{3}{8}$ when $\cos ^{2} \theta=\frac{1}{4}$.

As implied from the above consideration, the pure product states which saturate the maximum value $\frac{3}{8}$ are of the form

$$
|\gamma\rangle=|\alpha\rangle \otimes|\beta\rangle=\frac{\sqrt{3}}{2}\left(\begin{array}{c}
\cos \varphi \\
e^{i \delta_{1}} \sin \varphi \\
\frac{1}{\sqrt{3}} e^{i \delta_{2}}
\end{array}\right) \otimes \frac{\sqrt{3}}{2}\left(\begin{array}{c}
\cos \varphi \\
e^{i \delta_{1}} \sin \varphi \\
\frac{1}{\sqrt{3}} e^{i \delta_{2}}
\end{array}\right)
$$


in which $\delta_{1}$ and $\delta_{2}$ are arbitrary real phases.

\section{The proof of (4.20):}

We have

$$
\begin{aligned}
& \frac{3}{4}\left(P_{1}+P_{2}+P_{1,2}+P_{2,1}+P_{3}+P_{4}+P_{5}+P_{4,5}+P_{5,4}+P_{6}+P_{7}+P_{6,7}+P_{7,6}+P_{8}\right)-\frac{7}{4} \\
= & 3\left[\operatorname{Re}\left(\alpha_{0}^{*} \alpha_{1}\right) \operatorname{Re}\left(\beta_{0}^{*} \beta_{1}\right)+\operatorname{Im}\left(\alpha_{0}^{*} \alpha_{1}\right) \operatorname{Im}\left(\beta_{0}^{*} \beta_{1}\right)+\operatorname{Re}\left(\alpha_{0}^{*} \alpha_{1}\right) \operatorname{Im}\left(\beta_{0}^{*} \beta_{1}\right)+\operatorname{Im}\left(\alpha_{0}^{*} \alpha_{1}\right) \operatorname{Re}\left(\beta_{0}^{*} \beta_{1}\right)\right. \\
& +\operatorname{Re}\left(\alpha_{0}^{*} \alpha_{2}\right) \operatorname{Re}\left(\beta_{0}^{*} \beta_{2}\right)+\operatorname{Im}\left(\alpha_{0}^{*} \alpha_{2}\right) \operatorname{Im}\left(\beta_{0}^{*} \beta_{2}\right)+\operatorname{Re}\left(\alpha_{0}^{*} \alpha_{2}\right) \operatorname{Im}\left(\beta_{0}^{*} \beta_{2}\right)+\operatorname{Im}\left(\alpha_{0}^{*} \alpha_{2}\right) \operatorname{Re}\left(\beta_{0}^{*} \beta_{2}\right) \\
& \left.+\operatorname{Re}\left(\alpha_{1}^{*} \alpha_{2}\right) \operatorname{Re}\left(\beta_{1}^{*} \beta_{2}\right)+\operatorname{Im}\left(\alpha_{1}^{*} \alpha_{2}\right) \operatorname{Im}\left(\beta_{1}^{*} \beta_{2}\right)+\operatorname{Re}\left(\alpha_{1}^{*} \alpha_{2}\right) \operatorname{Im}\left(\beta_{1}^{*} \beta_{2}\right)+\operatorname{Im}\left(\alpha_{1}^{*} \alpha_{2}\right) \operatorname{Re}\left(\beta_{1}^{*} \beta_{2}\right)\right] \\
+ & \frac{3}{4}\left(\left|\alpha_{0}\right|^{2}-\left|\alpha_{1}\right|^{2}\right)\left(\left|\beta_{0}\right|^{2}-\left|\beta_{1}\right|^{2}\right)+\frac{1}{4}\left(1-3\left|\alpha_{2}\right|^{2}\right)\left(1-3\left|\beta_{2}\right|^{2}\right)-\frac{7}{4} \\
= & 3\left|\alpha_{0}\right|\left|\alpha_{1}\right|\left|\beta_{0}\right|\left|\beta_{1}\right|\left(\cos \left(\theta_{01}-\theta_{01}^{\prime}\right)+\sin \left(\theta_{01}+\theta_{01}^{\prime}\right)\right)+3\left|\alpha_{0}\right|\left|\alpha_{2}\right|\left|\beta_{0}\right|\left|\beta_{2}\right|\left(\cos \left(\theta_{02}-\theta_{02}^{\prime}\right)\right. \\
+3 \mid \alpha_{0} & || \alpha_{2}|| \beta_{0}|| \beta_{2}\left|\left(\cos \left(\theta_{02}-\theta_{02}^{\prime}\right)+\sin \left(\theta_{02}+\theta_{02}^{\prime}\right)\right)+3\right| \alpha_{1}|| \alpha_{2}|| \beta_{1}|| \beta_{2} \mid\left(\cos \left(\theta_{12}-\theta_{12}^{\prime}\right)+\sin \left(\theta_{12}+\theta_{12}^{\prime}\right)\right) \\
+ & \frac{3}{4}\left(\left|\alpha_{0}\right|^{2}-\left|\alpha_{1}\right|^{2}\right)\left(\left|\beta_{0}\right|^{2}-\left|\beta_{1}\right|^{2}\right)+\frac{1}{4}\left(1-3\left|\alpha_{2}\right|^{2}\right)\left(1-3\left|\beta_{2}\right|^{2}\right)-\frac{7}{4} \\
& \leq 6\left|\alpha_{2}\right|\left|\alpha_{2}\right|\left(\left|\alpha_{0}\right|\left|\beta_{0}\right|+\left|\alpha_{1}\right|\left|\beta_{1}\right|+\left|\alpha_{2}\right|\left|\beta_{2}\right|\right)-\frac{15}{4}\left|\alpha_{2}\right|^{2}\left|\beta_{2}\right|^{2}+\frac{3}{4}\left(\left|\alpha_{0}\right|\left|\beta_{0}\right|+\left|\alpha_{1}\right|\left|\beta_{1}\right|\right)^{2} \\
& \quad-\frac{3}{4}\left(\left|\alpha_{0}\right|\left|\beta_{1}\right|-\left|\alpha_{1}\right|\left|\beta_{0}\right|\right)^{2}-\frac{3}{4}\left(\left|\alpha_{2}\right|^{2}+\left|\beta_{2}\right|^{2}\right)-\frac{3}{2} .
\end{aligned}
$$

By using the Cauchy-Schwartz inequality, we have

$$
\leq 6\left|\alpha_{2}\right|\left|\alpha_{2}\right|-\frac{15}{4}\left|\alpha_{2}\right|^{2}\left|\beta_{2}\right|^{2}+\frac{3}{4}\left[\left(\left|\alpha_{0}\right|\left|\beta_{0}\right|+\left|\alpha_{1}\right|\left|\beta_{1}\right|\right)^{2}-\left(\left|\alpha_{0}\right|\left|\beta_{1}\right|-\left|\alpha_{1}\right|\left|\beta_{0}\right|\right)^{2}\right]-\frac{3}{4}\left(\left|\alpha_{2}\right|^{2}+\left|\beta_{2}\right|^{2}\right)-\frac{3}{2} .
$$

The parametrization ( $(\underline{\mathrm{I}-3})$ yields

$$
=6 \cos \theta \cos \theta^{\prime}-\frac{15}{4} \cos ^{2} \theta \cos ^{2} \theta^{\prime}+\frac{3}{4}\left[\sin ^{2} \theta \sin ^{2} \theta^{\prime} \cos 2\left(\varphi-\varphi^{\prime}\right)-\cos ^{2} \theta-\cos ^{2} \theta^{\prime}\right]-\frac{3}{2} .
$$

Since the Cauchy-Schwartz inequality becomes an equality when the two vectors are parallel, we must have $\theta=\theta^{\prime}$ and $\varphi=\varphi^{\prime}$. Then

$$
\leq 3 \cos ^{2} \theta-\cos ^{4} \theta-\frac{3}{4}
$$

The maximum value of the latter expression is 0 which come from $\cos ^{2} \theta=\frac{1}{2}$.

\section{Appendix III :}

\section{Proving the (3.15):}


To prove (3.15), we try to find all pure states that are orthogonal to all pure product states (I-4). To this aim, let us consider a pure state as

$$
|\psi\rangle=\sum_{i, j=0}^{2} a_{i j}|i j\rangle .
$$

and try to find the coefficients $a_{i j}$ such that $|\psi\rangle$ be orthogonal to all pure product states (I-4). We note that

$$
\begin{gathered}
\langle\gamma \mid \psi\rangle=a_{00}\left(3 \cos ^{2} \varphi\right)+a_{01}\left(3 e^{-i \delta_{1}} \sin \varphi \cos \varphi\right)+a_{02}\left(\sqrt{3} e^{-i \delta_{2}} \cos \varphi\right)+a_{10}\left(3 e^{-i \delta_{1}} \sin \varphi \cos \varphi\right) \\
+a_{11}\left(3 e^{-2 i \delta_{1}} \sin ^{2} \varphi\right)+a_{12}\left(\sqrt{3} e^{-i\left(\delta_{1}+\delta_{2}\right)} \sin \varphi\right)+a_{20}\left(\sqrt{3} e^{-i \delta_{2}} \cos \varphi\right) \\
+a_{21}\left(\sqrt{3} e^{-i\left(\delta_{1}+\delta_{2}\right)} \sin \varphi\right)+a_{22} e^{-i\left(\delta_{1}+\delta_{2}\right)}
\end{gathered}
$$

For $\varphi=0$, we get

$$
3 a_{00}+\sqrt{3} e^{-i \delta_{2}}\left(a_{02}+a_{20}\right)+a_{22} e^{-i\left(\delta_{1}+\delta_{2}\right)}=0
$$

and arbitrariness of phases $\delta_{1}$ and $\delta_{2}$ follows that

$$
a_{00}=a_{22}=0 \quad, \quad a_{20}=-a_{20} .
$$

$\varphi=\frac{\pi}{2}$ yields

$$
3 a_{11} e^{-2 i \delta_{1}}+\sqrt{3} e^{-i\left(\delta_{1}+\delta_{2}\right)}\left(a_{12}+a_{21}\right)=0
$$

and, as before, arbitrariness of phases $\delta_{1}$ and $\delta_{2}$ follows that

$$
a_{11}=0 \quad, \quad a_{21}=-a_{12} .
$$

Substituting the above results in ( $(\overline{\mathbb{I}-5})$, we have $a_{10}=-a_{01}$. Finally, it is concluded that the mutually orthogonal pure states

$$
\left|\psi_{1}\right\rangle=\frac{1}{\sqrt{2}}(|01\rangle-|10\rangle) \quad, \quad\left|\psi_{2}\right\rangle=\frac{1}{\sqrt{2}}(|02\rangle-|20\rangle) \quad, \quad\left|\psi_{3}\right\rangle=\frac{1}{\sqrt{2}}(|12\rangle-|21\rangle)
$$

are orthogonal to all pure product states $(\underline{\mathrm{I}-4})$ and the positive operator $\mathcal{P}$ in (3.15) is

$$
\mathcal{P}=\frac{27}{4}\left|\psi_{1}\right\rangle\left\langle\psi_{1}\right|+\frac{3}{4}\left(\left|\psi_{2}\right\rangle\left\langle\psi_{2}|+| \psi_{3}\right\rangle\left\langle\psi_{3}\right|\right)
$$

Now, we consider another pure state as

$$
|\varphi\rangle=\sum_{i, j=0}^{2} b_{i j}|i j\rangle .
$$


and try to find the coefficients $b_{i j}$ such that $|\varphi\rangle$ be orthogonal to all pure product states $\left|\alpha^{*}\right\rangle \otimes|\beta\rangle$ in (I-4). By similar arguments as above, we conclude that

$$
|\varphi\rangle=\frac{1}{\sqrt{11}}(|00\rangle+|11\rangle-3|22\rangle)
$$

and

$$
\mathcal{Q}=\frac{33}{8}|\varphi\rangle\langle\varphi|
$$

\section{References}

[1] M. A. Nielsen and I. L. Chuang, Quantum Computation and Quantum Information (Cambridge University Press, Cambridge, 2000).

[2] The Physics of Quantum Information: Quantum Cryptography, Quantum Teleportation and Quantum Computation, edited by D. Bouwmeester, A. Ekert, and A. Zeilinger (Springer, New York, 2000).

[3] J. Preskill, The Theory of Quantum Information and Quantum Computation (California Inatitute of Technology, Pasadena, CA, 2000), http://www.theory.caltech.edu/poeole/preskill/ph229/.

[4] B. Baumgartner, B. C. Hiesmayr, H. Narnhofer, Phys. Rev. A 74, 032327 (2006).

[5] B. Baumgartner, B. C. Hiesmayr, H. Narnhofer, e-print: quant-ph/0705.1403 (2007).

[6] R. A. Bertlmann, K. Durstberger, B. C. Hiesmayr, P. Krammer, Phys. Rev. A 72, 052331 (2005).

[7] A.B. Klimov, L.L. Snchez-Soto, H. de Guise, G. Bjrk, J. Phys. A: Math. Gen. 37, 4097-4106 (2004).

[8] S. L. Woronowicz, Rep. on Math. Phys. 10, 165 (1976).

[9] M. Horodecki, P. Horodecki, and R. Horodecki, Phys. Lett. A 223, 1 (1996).

[10] A. C. Doherty, P. A. Parrilo, and F. M. Spedalieri, Phys. Rev. A 71, 032333 (2005). 
[11] R. O. Vianna, A. C. Doherty, Phys. Rev. A, 74, 052306 (2006).

[12] M. A. Jafarizadeh, M. Rezaee, S. K. A. Seyed Yagoobi, Phys. Rev. A 72, 062106 (2005).

[13] M. A. Jafarizadeh, M. Rezaee, S. Ahadpour, Phys. Rev. A 74, 042335 (2006).

[14] M. A. Jafarizadeh, G. Najarbashi, H. Habibian, Phys. Rev. A 75, 052326 (2007).

[15] M. A. Jafarizadeh, G. Najarbashi, Y. Akbari, H. Habibian, e-print: quant-ph/0704.2414 (2007)(accepted for publication in EPJD).

[16] M. A. Jafarizadeh, R. Sufiani, Phys. Rev. A 77, 012105 (2008).

[17] S. Boyd and L. Vandenberghe, Convex Optimization, Cambridge University Press (2004).

[18] E. K. P. Chong and S. H. Żak, An Introduction to Optimization, John Wiley, NY (2001).

[19] W. Pfeifer, The Lie Algebras su(N) An Introduction, Birkhäuser Verlag (2003).

[20] W. Rudin, Functional Analysis,(McGraw-Hill, Singapore,1991).

[21] M. Lewenstein, D. Bruß, J.I. Cirac, B. Kraus, M. Kus, J. Samsonowicz, A. Sanpera, and R. Tarrach, J. Mod. Opt. 47, 2841 (2000).

[22] R. A. Bertlmann, and P. Krammer, e-print: quant-ph/0706.1743v1 (2007).

[23] M. Horodecki, P. Horodecki, and R. Horodecki, Phys. Rev. Lett 82, 1056 (1999).

[24] A. C. Doherty, P. A. Parrilo, and F. M. Spedalieri, Phys. Rev. Lett. 88, 187904 (2002). 